\title{
THE DEVELOPMENT OF CEREBRAL AMYLOID ANGIOPATHY IN CEREBRAL VESSELS. A REVIEW WITH ILLUSTRATIONS BASED UPON OWN INVESTIGATED POST MORTEM CASES
}

\author{
Tadeusz Andrzej Mendel, Teresa Wierzba-Bobrowicz, Eliza Lewandowska, Tomasz Stępień, \\ GRAŻYNA MARIA SZPAK
}

Institute of Psychiatry and Neurology, Warsaw, Poland

\begin{abstract}
The process of $\beta$-amyloid accumulation in cerebral vessels is presented. Cerebral amyloid angiopathy (CAA) was confirmed during an autopsy. It was diagnosed according to the Boston criteria. Cerebral amyloid angiopathy can involve all kinds of cerebral vessels (cortical and leptomeningeal arterioles, capillaries and veins). The development of CAA is a progressive process. $\beta$-amyloid appears first in the tunica media, surrounding smooth muscle cells, and in the adventitia. $\beta$-amyloid is progressively accumulated, causing a gradual loss of smooth muscle cells in the vessel wall and finally replacing them. Then, the detachment and delamination of the outer part of the tunica media results in the "double barrel" appearance, fibrinoid necrosis, and microaneurysm formation. Microbleeding with perivascular deposition of erythrocytes and blood breakdown products can also occur. $\beta$-amyloid can also be deposited in the surrounding of the affected vessels of the brain parenchyma, known as "dysphoric CAA". Ultrastructurally, when deposits of amyloid fibers were localized in or outside the arteriolar wall, the degenerating vascular smooth muscle cells were observed. In the Institute of Psychiatry and Neurology the study was carried out in a group of 48 patients who died due to intracerebral hemorrhage caused by sporadic CAA.
\end{abstract}

Key words: celebral amyloid angiopathy, CAA, $\beta$-amyloid, intracelebral hemorrhage, ultrastructure.

\section{Introduction}

Cerebral amyloid angiopathy (CAA) refers to deposition of congophilic material in the cerebral vasculature, particularly small and medium-sized arteries, capillaries and veins of leptomeninges and the cerebral cortex [1-9] (Fig. 1).

The amyloid peptide contained in vascular deposits of CAA is identical to the $\beta$-amyloid found in senile plaques in Alzheimer's disease [4].

Cerebral amyloid angiopathy occurs mostly as a sporadic condition in the elderly, with an incidence associated with increasing age. It is recognized as a cause of lobar intracerebral hemorrhage.

Neuropathological examination is still a "gold standard" for CAA diagnosis. Using the Boston crite- ria for CAA diagnosis and having CT and/or MRI scans the neurologist may select those of the patients whom he or she most suspects of having CAA [10]. However, the definite diagnosis of CAA can be made through a postmortem examination or an intracranial biopsy $[1,10,11]$.

Cerebral amyloid angiopathy can be divided into sporadic and familiar forms. Sporadic CAA occurs much more frequently and typically in older patients. It occurs in equal frequency in men and women [12].

In one of the first autopsy studies, among people over 60 years of age without dementia, 30\% were found to have CAA, while the prevalence of CAA with a pathologically certain Alzheimer syndrome was $82 \%$ [4]. In another study, among patients suffering from dementia an average of $55-59 \%$ suffered from CAA, while a CAA 

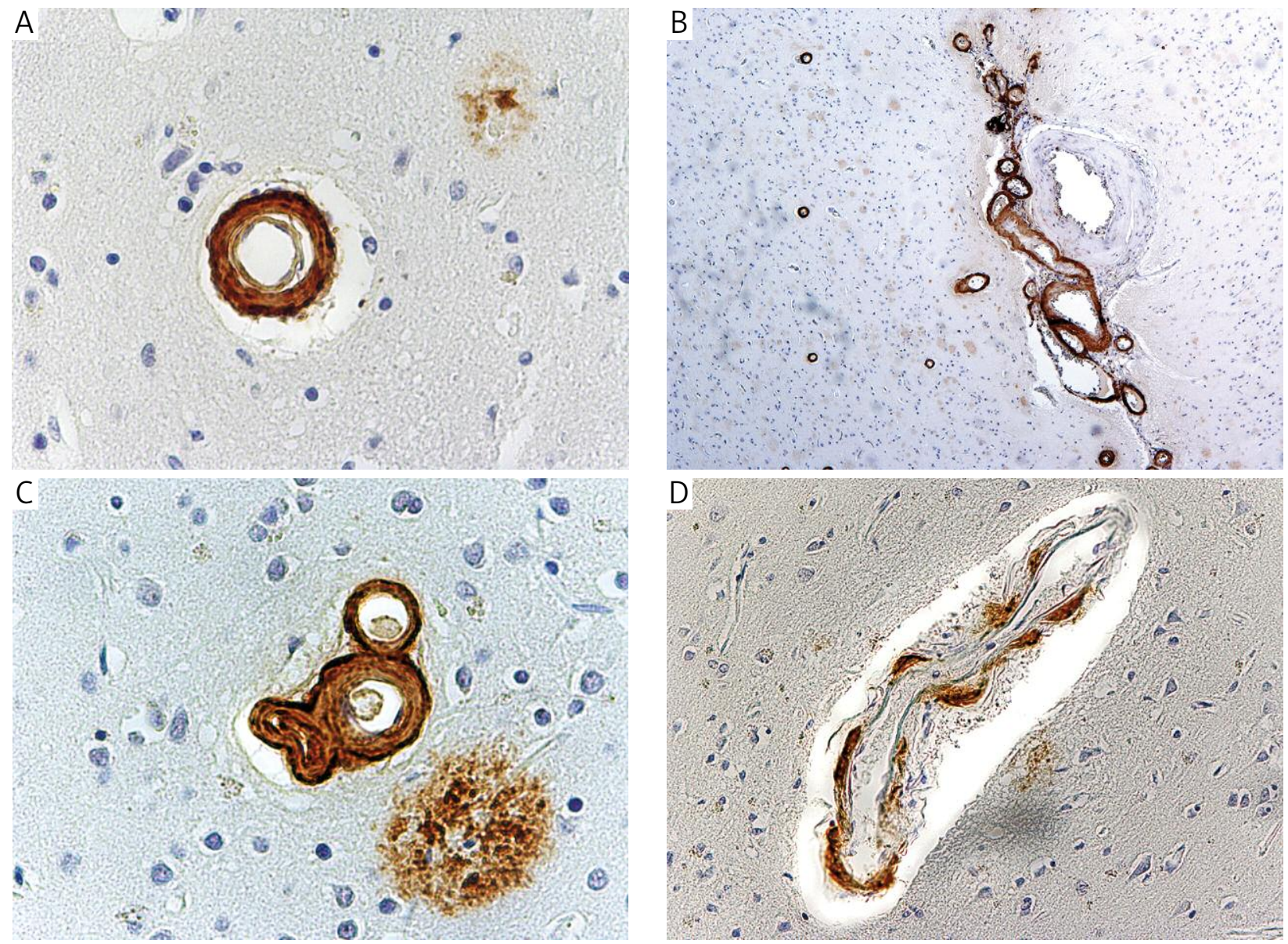

Fig. 1. A) $\beta$-amyloid deposits in cortical vessel. Anti-A $\beta 400 \times$. B) $\beta$-amyloid deposits in leptomeningeal vessels. Anti-A $\beta 100 \times$. C) $\beta$-amyloid deposits in capillaries. Anti-A $\beta 400 \times$. D) $\beta$-amyloid deposits in veins. Anti-A $\beta 200 \times$

prevalence of only $28-38 \%$ was determined in patients without dementia [13].

The only genetic risk factors known for sporadic CAA are apolipoprotein $\mathrm{E}$ (ApoE) alleles [12]. The $\varepsilon 2$ allele favors CAA-related vascular deterioration (splitting of vascular walls, minor hemorrhaging and fibrinoid necrosis), while the $\varepsilon 4$ allele is associated with increased vascular deposition of $A \beta$. Other genetic polymorphisms, such as presenilin 1, $\alpha 1$ antichymotrypsin, neprilysin, oxidized LDL receptors, lipoprotein receptor-related protein, interleukin 33 and transforming growth factor $\beta 1$, are discussed as possible etiological factors of CAA [12].

The Vonsattel, Mountjoy and Olichney scales are used to assess the grade and scores of $\beta$-amyloid deposited in vessel walls [14-16].

On microscopic evaluation the brain is considered positive for CAA when it shows at least one leptomeningeal or cortical congophilic vessel with yellowgreen birefringence under polarized light and/or $\beta$-amyloid deposits seen within the wall of vessels. The severity of CAA was classified according to the Vonsattel scale as follows: 1 . Mild, when $\beta$-amyloid is restricted to a congophilic rim around normal or atrophic smooth muscle fibers (which might be absent), leaving an op- tically empty tiny vacuole-like zone surrounded by amyloid in the media of otherwise normal vessels. 2. Moderate, when the media is replaced by $\beta$-amyloid and is thicker than normal, with no evidence of remote or recent blood leakage. 3. Severe, when there is extensive $\beta$-amyloid deposition with focal wall fragmentation and at least one focus of perivascular leakage evidenced by the presence of erythrocytes or hemosiderin or both [16].

The estimated proportion of $\beta$-amyloid involvement in each vessel was recorded on the Mountjoy scale of 0-4. A score of 0 indicated the absence of amyloid; a score of 1 , involvement of up to one-quarter of the vessel circumference; 2 , involvement of up to one-half of the vessel circumference; 3 , involvement of up to three-quarters of the vessel circumference; and a score of 4 indicated total involvement of the vessel [14].

According to the Olichney scale of 0-4 the severity of cerebral amyloid angiopathy was classified as follows: A score of 0 meant no thioflavin-S positivity in the leptomeningeal and superficial cortical blood vessels. A score of 1 reflected traces of scattered positivity in either leptomeningeal or cortical blood vessels. A score of 2 indicated that at least some vessels in the leptomeninges or neocortex had circumferential bright- 
ly staining amyloid deposits. A score of 3 indicated widespread circumferential thioflavin-S positivity in many leptomeningeal and superficial cortical vessels. A score of 4 indicated that the similarly severe amyloid angiopathy was combined with dysphoric changes, i.e., thioflavin-S positivity emanating from severely amyloid blood vessels into surrounding neuropil [15].

There are a lot of diagnostic tools which can be used in the diagnosis of CAA during intracerebral hemorrhage, but the neuropathological examination can only confirm this disease and reveal all stages of CAA. It can also evidence all kinds of vessels involved in this process and allow the level of CAA and its localization to be classified $[1,10,11,17]$.

\section{Clinical symptomatology}

Cerebral amyloid angiopathy can be clinically manifested as intracranial hemorrhage (lobar or minor hemorrhaging), ischemic stroke, leukoencephalopathy, transient ischemic attack, dementia, vasculitis or tumor [2, 11, 18-24].

Cerebral amyloid angiopathy often appears in elderly patients though symptomatic, spontaneous lobar hemorrhaging evoking a neurological focal deficit, headaches, impairment of consciousness, seizures, cognitive impairments, dementia, depression, behavioral problems and personality changes $[2,11,18-24]$.

\section{Diagnosis}

Brain MRI, PET with use of Pittsburgh compound $\mathrm{B}$, and cerebrospinal fluid examination with assessment of the concentration of $A \beta 1-40, A \beta 1-42$ and tau pro- teins are tools used in CAA diagnosis. Also endoscopy of the ocular fundus showing retinal changes as well as spot- or dot-shaped hemorrhaging are helpful in CAA diagnosis. The neuroimaging symptoms of CAA can lead to intracerebral hemorrhages mainly located in posterior brain regions and to superficial siderosis.

Although CAA can be detected pathologically for over $80 \%$ of patients with a pathologically certain Alzheimer syndrome [25], less than $50 \%$ of patients suffering from a pathologically certain CAA present the pathological criteria for the Alzheimer syndrome [17, 26].

\section{Pathogenesis of cerebral amyloid angiopathy}

The pathogenesis of CAA remains unclear, and the source of $\beta$-amyloid has not been definitely elucidated. There are several theories proposed by different groups of researchers. $\beta$-amyloid may originate from blood, from perivascular space or from cerebrospinal fluid, or may be produced by smooth muscle cells and by neurons [11, 27-31].

Some researchers suggest that a soluble precursor of $\beta$-amyloid, for instance immunoglobulins or apolipoproteins E, may originate from blood and can be transported with assistance of the protein receptor through an impaired blood-brain barrier, inducing the accumulation of insoluble $\beta$-amyloid in brain vessels and brain tissues [32, 33].

Another hypothesis assumes that smooth muscle cells localized in vessels or pericytes are the source of $\beta$-amyloid $[23,28,34]$. $\beta$-amyloid can be produced by smooth muscle cells as a consequence of vessel wall damage probably due to atherosclerosis and/or hypertension [7, 23, 28, 34-38].

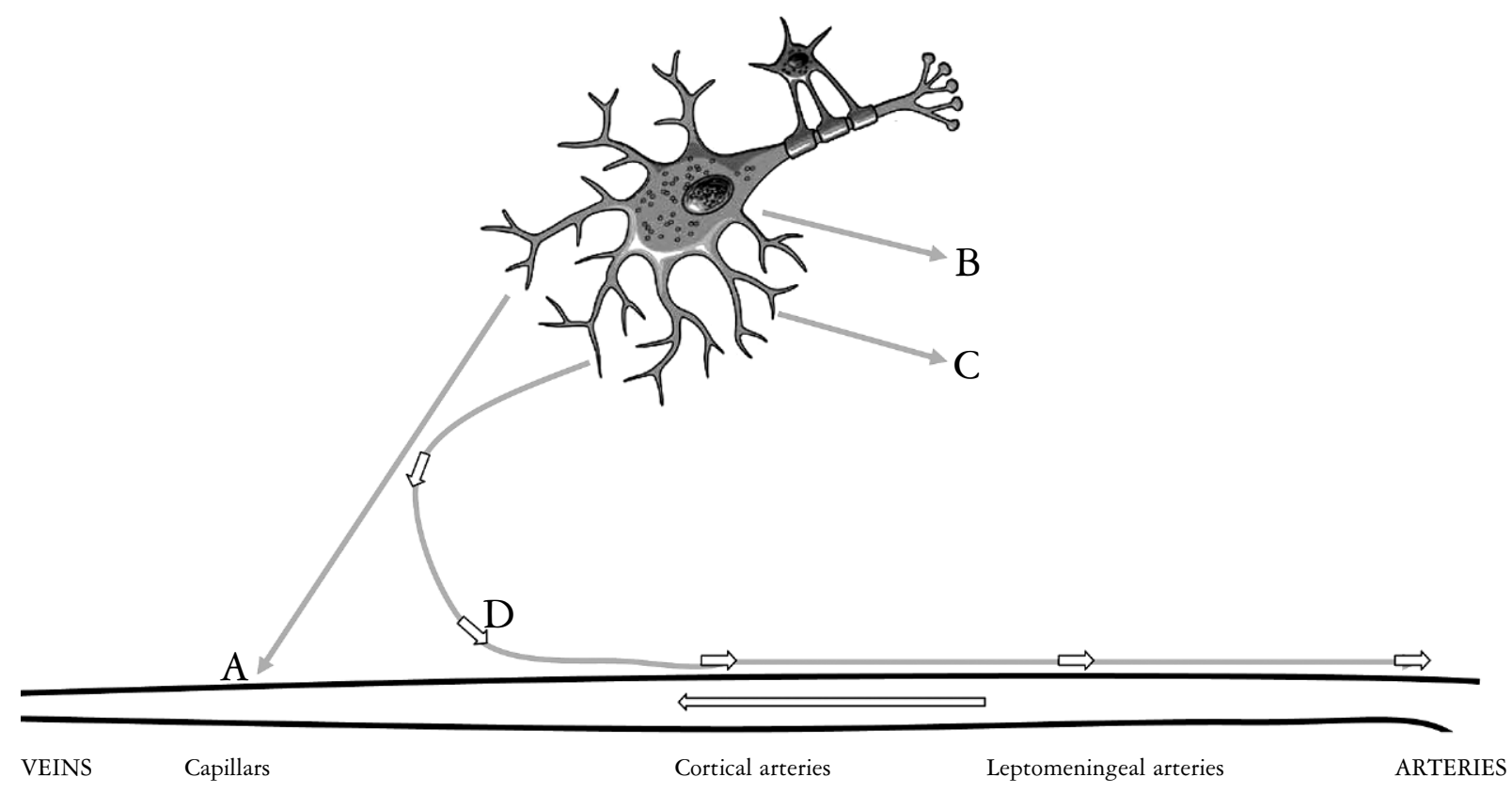

Fig. 2. Different pathways for $\beta$-amyloid elimination from the brain modified according Weller $[8,9]$ 
Still another hypothesis suggests that $\beta$-amyloid is of neuronal origin and it is transported via interstitial fluid drainage pathways from the brain among basement membranes of the capillary and artery walls, using effectively the perivascular lymphatic drainage pathway from the brain to the blood vessels $[3,7-9,28$, 35-37, 39-41].

There are several pathways of $\beta$-amyloid elimination from the brain (Fig. 2). The imbalance between $\beta$-amyloid production and clearance (degradation and elimination of $\beta$-amyloid from the brain) is generally considered a key element in the formation of amyloid deposits.

One of the pathway (A) of elimination via brain vessels by a mechanism mediated by low-density lipoprotein is lipoprotein-receptor related protein-1 (LRP-1) and it is degraded in the brain parenchyma by neprilysin and other enzymes. Among the receptors involved, the receptor for advanced glycation end-products actively participates in brain uptake of free $\beta$-amyloid at the level of vessel walls. Other receptors are more relevant for the transport of $\beta$-amyloid complexed with other molecules: LRP-1 mediates transcytosis of $\beta$-amyloid-ApoE complexes contributing to rapid brain clearance, whereas megalin mediates in the cellular uptake and transport of $\beta$-amyloid-ApoJ [6-9, 44-47, 49].

Another pathway (B) of $\beta$-amyloid elimination is done by microglia and astrocyte $[8,9]$.

Neprilysin, endothelin-amyloid-converting enzyme, insulin-degrading enzyme, $\beta$-amyloid-converting enzyme 1, plasmin and matrix metalloproteases are among major enzymes known to participate in brain $\beta$-amyloid catabolic pathways (C). Reduced levels and/or activity of $\beta$-amyloid degrading enzymes favor $\beta$-amyloid accumulation and increase the levels of $\beta$-amyloid deposits. The failure of LRP-1 and neprilysin mechanisms with increasing age can cause the failure of perivascular drainage of $\beta$-amyloid along stiff arteries. Aged arteries are associated with deposition of insoluble $\beta$-amyloid as plaques in brain parenchyma, and in blood walls as cerebral amyloid angiopathy $[6-9,29$, 40, 43-45, 47, 481.

$\beta$-amyloid can also be eliminated from the brain by the perivascular drainage $[8,39,40]$. The $\beta$-amyloid drains from the brain with interstitial fluid along capillary and artery walls (D). The amphiphilic nature of $\beta$-amyloid precludes its crossing through the bloodbrain barrier unless mediated by specialized carriers and/or receptor transport mechanisms. These mechanisms control the uptake of circulating $\beta$-amyloid into the brain and regulate clearance $[7,8,32,39,40]$.

\section{Neuropathology of vessels with $\beta$-amyloid deposits}

In the Institute of Psychiatry and Neurology the study was carried out in a group of 48 patients who died due to intracerebral hemorrhage as a result of CAA. All cases were sporadic CAA. In all cases autopsy was performed.

The autopsy was performed according to the standard protocol. The whole brain was fixed in $4 \%$ paraformaldehyde in $0.1 \mathrm{M}$ phosphate-buffered saline, embedded in paraffin and then sliced coronally. The frontal, temporal, parietal and occipital lobes were examined. The specimens were stained with Congo red and immunohistochemically with the following antibodies: anti-A $\beta$ 8-17 (DAKO, 1 : 50), actin (SMA, DAKO, 1 : 50) (Fig. 3).

For ultrastructural analysis, a small fragment of brain was taken from a paraffin block. After deparaffinizing and washing in tap water, brain specimens were fixed in $2.5 \%$ glutaraldehyde in cacodylate buffer and postfixed in $2 \%$ osmium tetroxide in the same buffer. They were then dehydrated and embedded in Epon. Ultrathin sections stained with uranyl acetate and lead citrate were examined using an Opto DPS 109 electron microscope.

There are three stages of $\beta$-amyloid accumulation in brain vessels according to the Vonsattel scale and
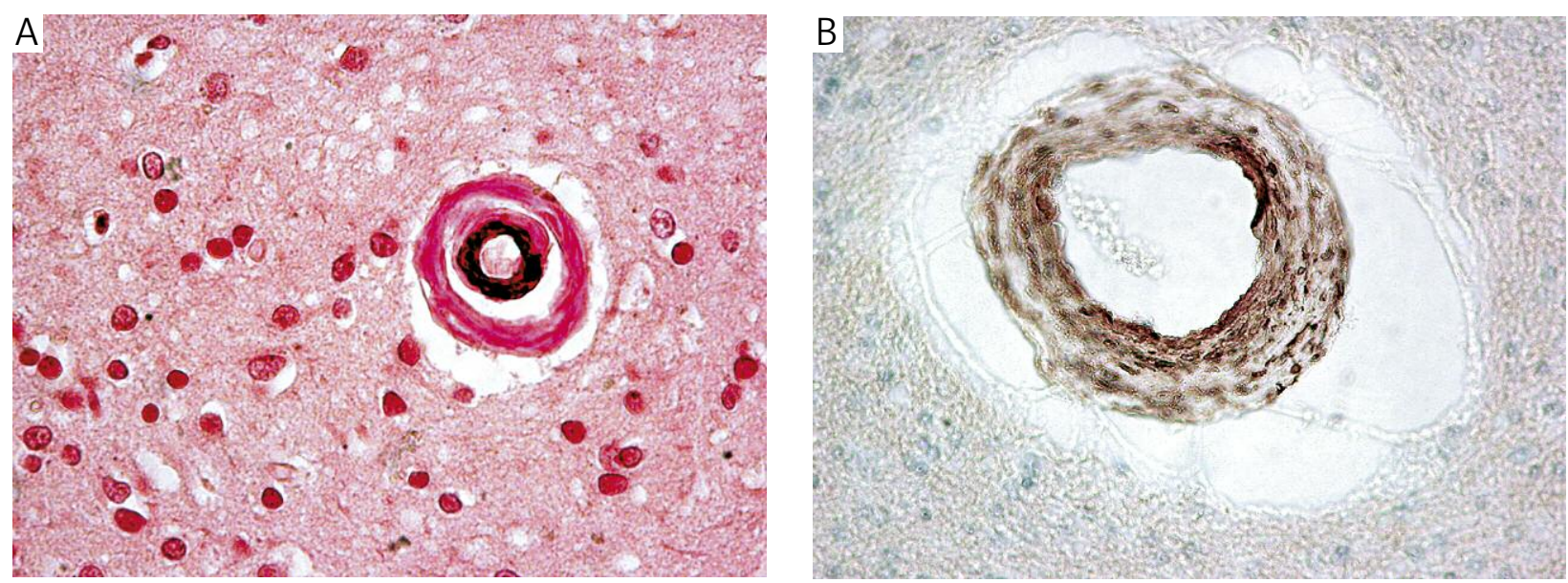

Fig. 3. Various stages of arterial CAA severity with "double barrel". Congo red, $200 \times$. CAA in arterial vessel. Actin, $400 \times$ 


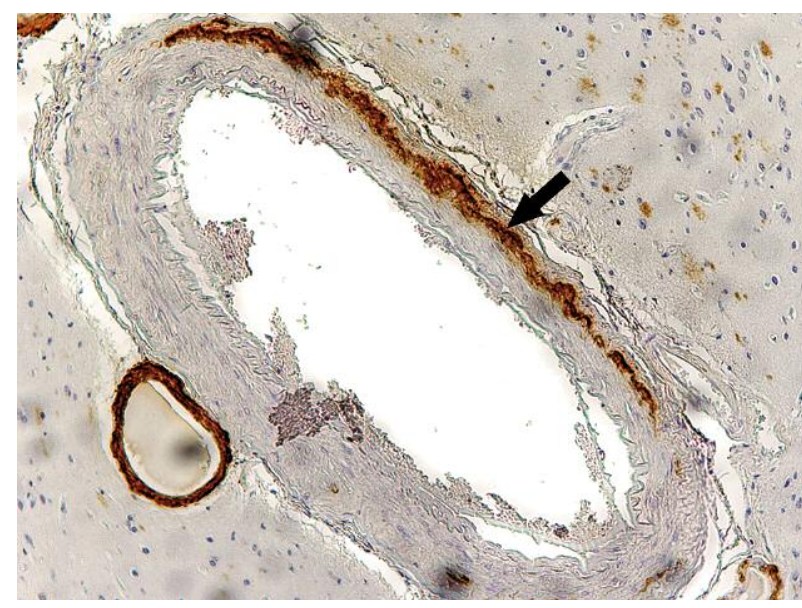

Fig. 4. $\beta$-amyloid deposits in the basement membrane (arrow). Anti-A $\beta, 400 \times$

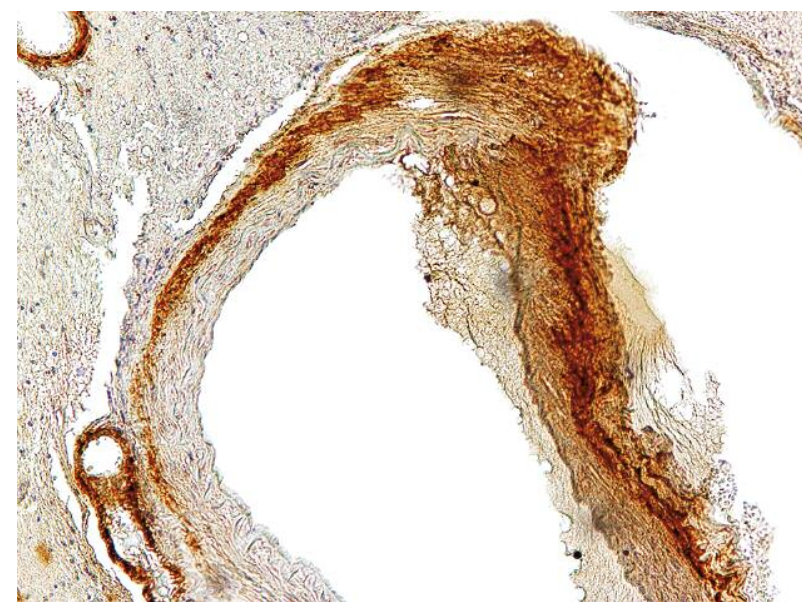

Fig. 5. $\beta$-amyloid deposits in the smooth muscle cells. Anti-A $\beta, 100 \times$

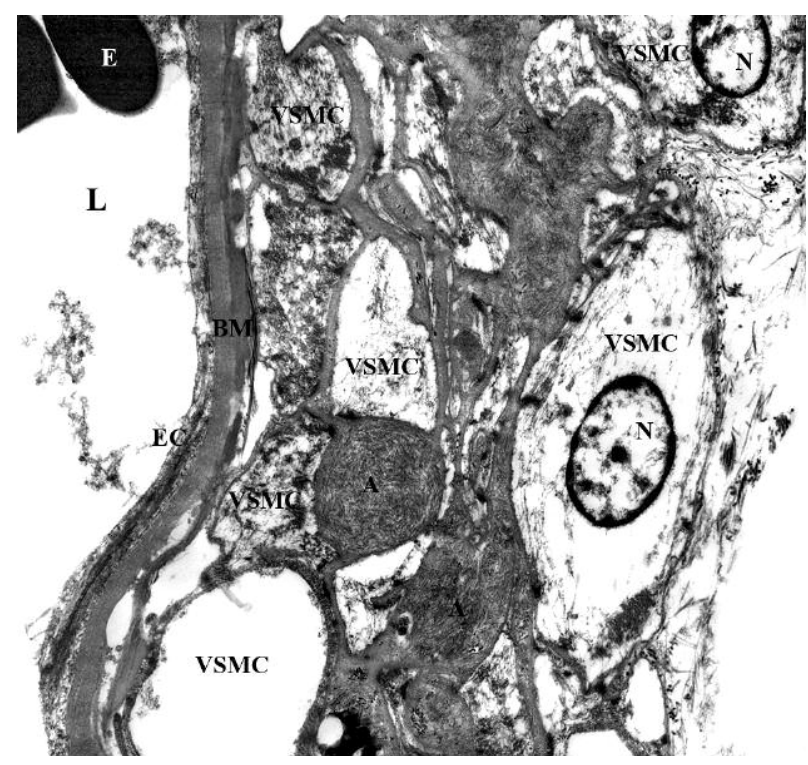

Fig. 6. Arteriolar wall with degenerative vascular smooth muscle cells (VSMC) and deposits of amyloid fibers (A) in basement membrane (BM). $\mathrm{N}$ - nucleus, $\mathrm{EC}$ - endothelial cell, L - lumen, $\mathrm{E}-$ erythrocytes. Original magnification $4400 \times$ four stages according to the Olichney and Mountjoy scales $[14-16]$.

First, $\beta$-amyloid $1-42$ starts to be deposited in vessels. Second, $\beta$-amyloid 1-40 begins to replace smooth muscle cells. This process takes place with coexistence of APOE $\varepsilon 4$. Third, the vessel wall is disrupted and APOE $\varepsilon 2$ contributes to the increase in this pathological process $[6,7,9,29,30,40,49]$.

Other results indicate that $\beta$-amyloid 1-40 is mainly localized in leptomeningeal arteries, arterioles and small cortical vessels, while both $\beta$-amyloid 1-40 and $\beta$-amyloid 1-42 are presented and $\beta$-amyloid 1-42 dominates in capillaries $[50,51]$.

The development of CAA is a progressive process. First, $\beta$-amyloid appears in the tunica media, surrounding smooth muscle cells, and in the adventitia (Fig. 4 and 5). Ultrastructurally, arteriolar wall with degenerative vascular smooth muscle cells and deposits of amyloid fibers in the basement membrane can be observed (Fig. 6). At the initial stage, the vessel wall structure was intact and $\beta$-amyloid was progressively accumulated, causing gradual loss of smooth muscle cells. $\beta$-amyloid was slowly and progressively replaced by the smooth muscle cells in the vessel wall (Figs. 7-10). Then, detachment and delamination of the outer part of the tunica media results in a "double barrel" appearance (Fig. 11), fibrinoid necrosis, and microaneurysm formation. Microbleeding with perivascular deposition of erythrocytes and blood breakdown products can also occur [3].

$\beta$-amyloid can also be deposited in the surrounding affected vessels of the brain parenchyma known as a "dysphoric CAA" (Fig. 12). Ultrastructurally, amyloid fibers expanding outside the ruptured vessel wall can be observed (Fig. 13) [3].

Deposits of $\beta$-amyloid in CAA were observed in all kinds of cerebral vessels: arteries (cortical and meningeal), capillaries and veins. Mostly in the same patients all kinds of vessels were intact but in those vessels that showed amyloid deposits the different stages of CAA were observed (Fig. 14).

Alzheimer's type pathology (neuritic plaques) Coexisted in 43\% with CAA (Fig. 15).

According to Weller, veins do not appear to be involved in CAA, although deposits of $\beta$-amyloid are found attached to the walls of veins [16]. Interestingly, Mendel et al. (2013) found the occurrence of $\beta$-amyloid even in $78 \%$ of veins [5].

\section{Conclusions}

Deposits of $\beta$-amyloid in CAA were observed in all kinds of cerebral vessels: arteries (cortical and meningeal), capillaries and veins. Neuropathological examination revealed that in the same patient different vessels contained all stages of CAA.

The study group consisted of patients with severe and lethal intracerebral hemorrhages and all kinds of vessels were involved. 


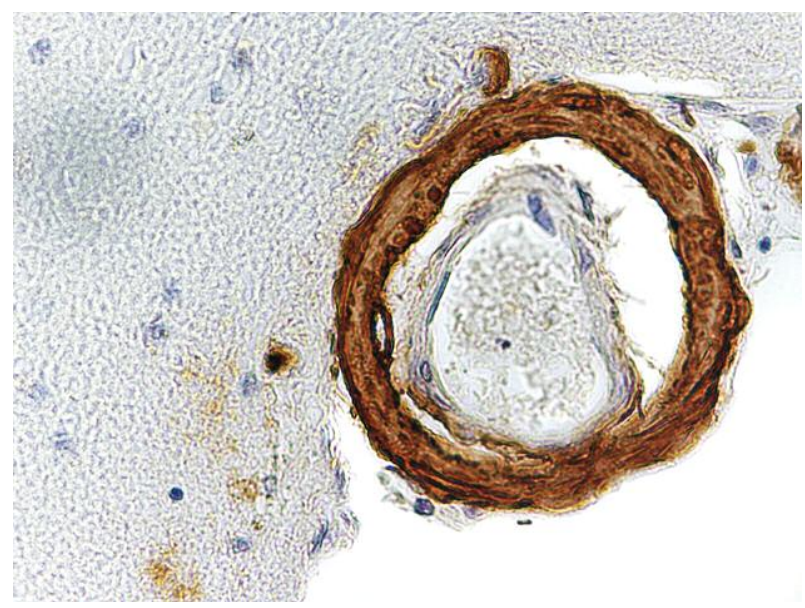

Fig. 7. $\beta$-amyloid deposits in the exfoliated endothelium in the cerebral artery "double-barrel". Anti-A $\beta, 400 \times$

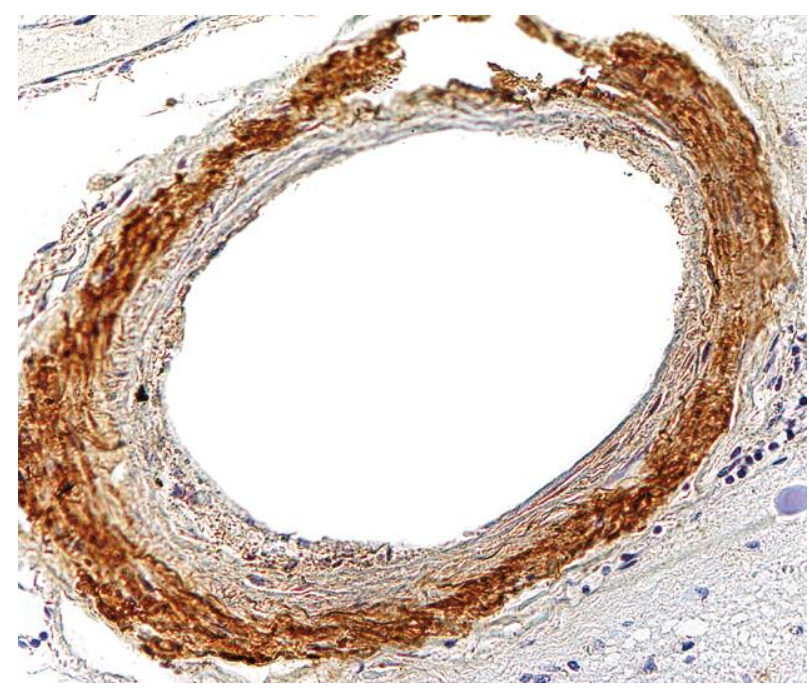

Fig. 9. Rupture of the vessel wall. Anti-A $\beta, 200 \times$

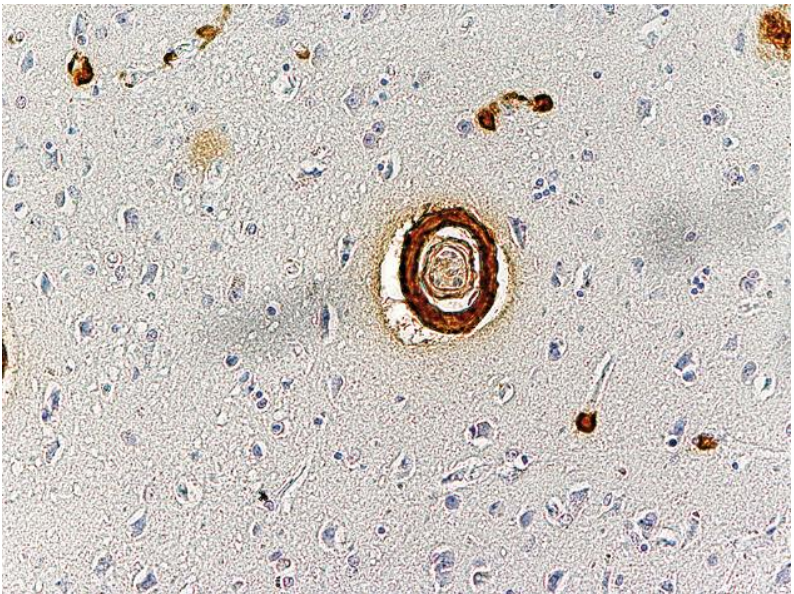

Fig. 11. "Double-barrel" of the wall in the cerebral cortical artery. Anti-A $\beta, 200 \times$

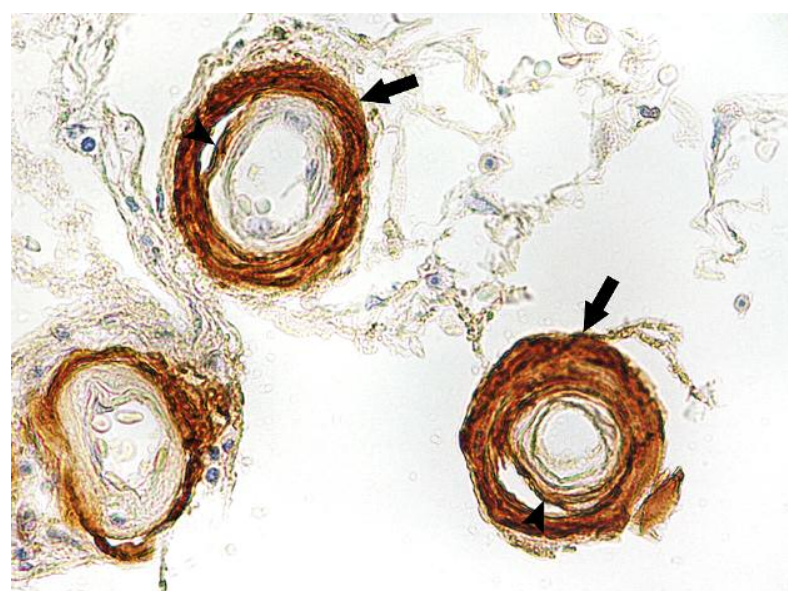

Fig. 8. $\beta$-amyloid deposits in the leptomeningeal artery (arrows) in one exfoliated endothelium are visible.

"Double-barrel" artery. Anti-A $\beta, 400 \times$

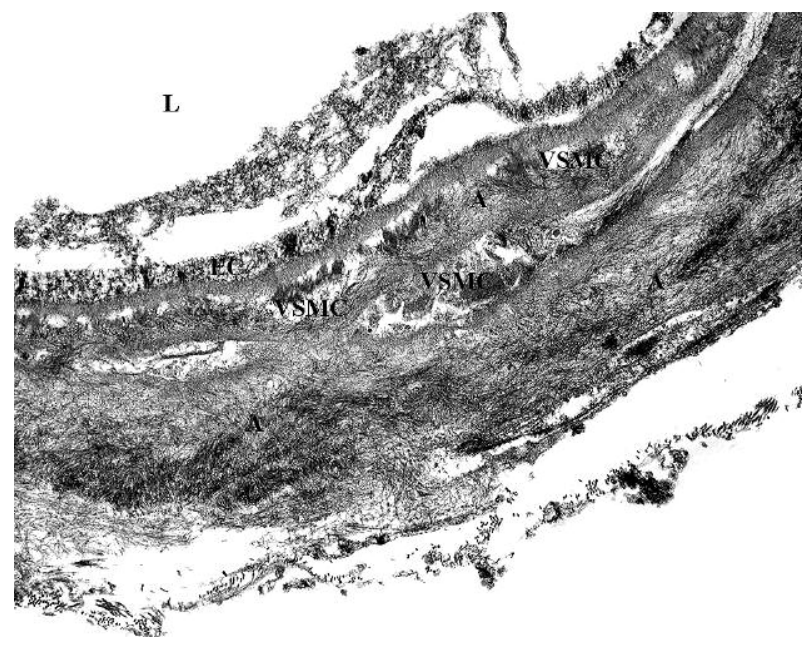

Fig. 10. Arteriolar wall with abundant amyloid fibers (A) around degenerative vascular smooth muscle debris (VSMC), EC - endothelial cell, L - lumen. Original magnification $4400 \times$

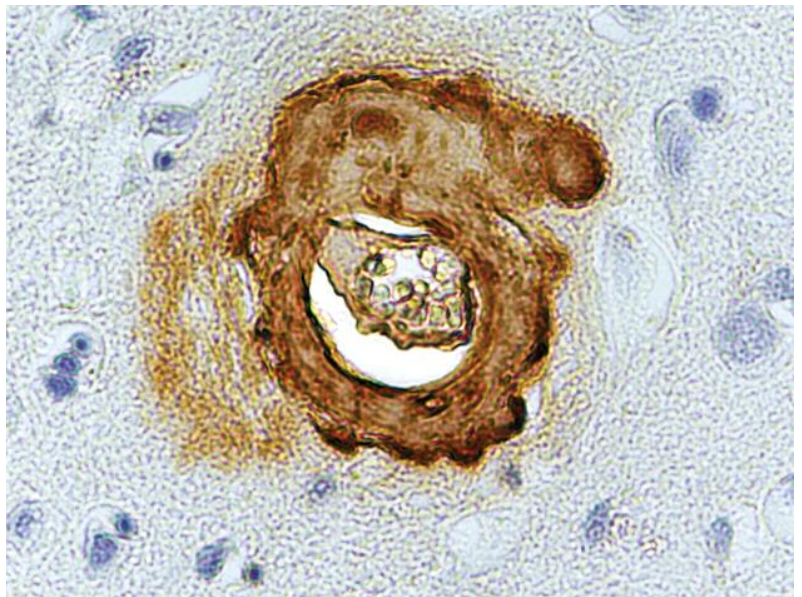

Fig. 12. $\beta$-amyloid deposits in the whole vessel and in surrounding brain parenchyma called "dysphoric CAA". Anti-A $\beta, 400 \times$ 


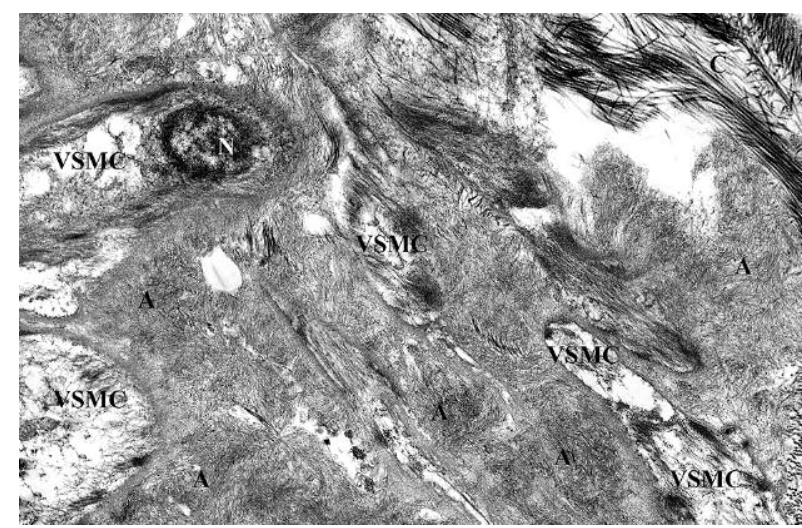

Fig. 13. Fragment of arteriolar wall with degeneration of vascular smooth muscle cells (VSMC). In the right upper corner, expansion of amyloid fibers (A) outside the arteriolar wall is visible. $\mathrm{C}$ - collagen fibers. Original magnification $4400 \times$

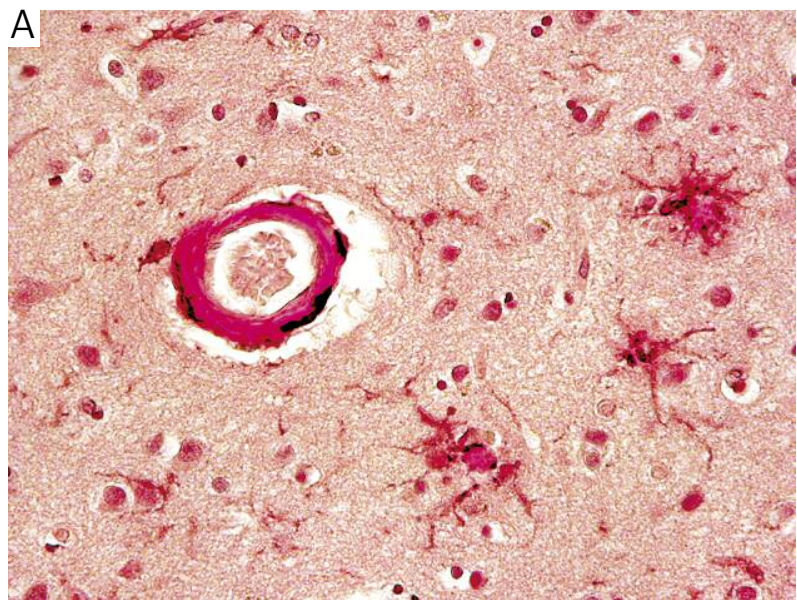

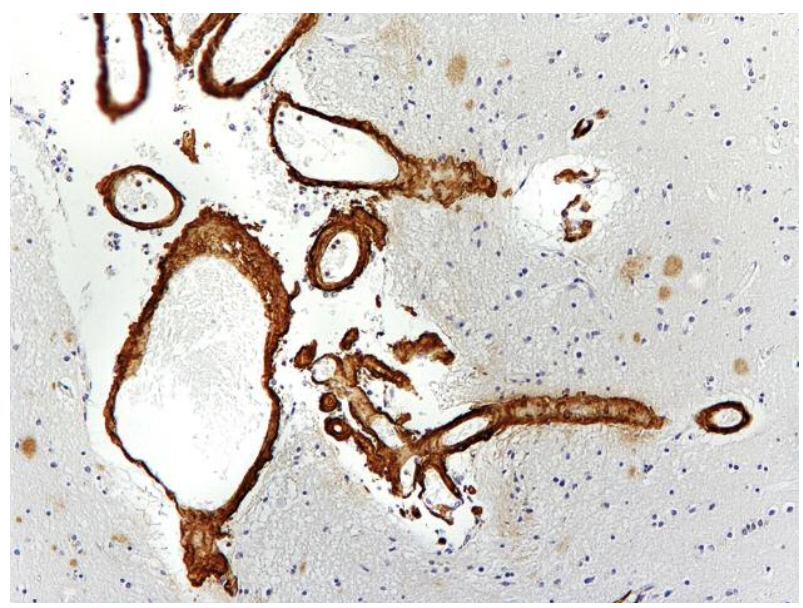

Fig. 14. $\beta$-amyloid deposits in all kinds of cerebral vessels. Anti-A $\beta, 100 \times$

Fig. 15. Alzheimer's type pathology. A) $\beta$-amyloid deposits in vessels and neuritic plaques. Congo red, $200 \times$.

B) Neuritic plaques and $\beta$-amyloid deposits in the wall of cortical vessels. Anti-A $\beta, 200 \times$

The development of CAA is a progressive and longterm process. $\beta$-amyloid slowly and effectively replaced the smooth muscle cells in cerebral vessel walls, causing damage and increasing the risk for intracerebral hemorrhage.

\section{References}

1. Attems J. Sporadic cerebral amyloid angiopathy: pathology, clinical implications, and possible pathomechanisms. Acta Neuropathol 2005; 110: 345-359.

2. Biffi A, Greenberg SM. Cerebral amyloid angiopathy: a systemic review. J Clin Neurol 2011; 7: 1-9.

3. Charidimou A, Gang Q, Werring DJ. Sporadic cerebral amyloid angiopathy revisited: recent insights into pathophysiology and clinical spectrum. J Neurol Neurosurg Psychiatry 2012; 83: 124-137.

4. Esiri MM, Wilcock GK. Cerebral amyloid angiopathy in dementia and old age. J Neurol Neurosurg Psychiatry 1986; 49: 12211226.

5. Mendel T, Wierzba-Bobrowicz T, Stępień T, Szpak GM. Vein's involvement by $\beta$-amyloid in intracerebral haemorrhage. Folia Neuropathol 2013; 51: 120-126.
6. Miners JS, Kehoe P, Love S. Neprilysin protects against cerebral amyloid angiopathy and $A \beta$-induced degeneration of cerebrovascular smooth muscle cells. Brain Pathol 2011; 21: 594-605.

7. Weller RO, Subash M, Preston SD, et al. Perivascular drainage of amyloid- $\beta$ peptides from the brain and its failure in cerebral amyloid angiopathy and Alzheimer's disease. Brain Pathol 2008; 18: 253-266

8. Weller RO, Djuanda E, Yow HY, Carare RO. Lymphatic drainage of the brain and the pathophysiology of neurological disease. Acta Neuropathol 2009; 117: 1-14.

9. Weller RO, Boche D, Nicoll JA. Microvasculature changes and cerebral amyloid angiopathy in Alzheimer's disease and their potential impact on therapy. Acta Neuropathol 2009; 118: 87-102.

10. Knudsen KA, Rosand J, Karluk D, Greenberg SM. Clinical diagnosis of cerebral amyloid angiopathy: validation of the Boston criteria. Neurology 2001; 56: 537-539.

11. Greenberg SM. Cerebral amyloid angiopathy: prospects for clinical diagnosis and treatment. Neurology 1998; 51: 690-694.

12. Gahr M, Nowak DA, Connemann BJ, Schönfeldt-Lecuona C. Cerebral amyloid angiopathy - a disease with implications for neurology and psychiatry. Brain Res 2013; 1519: 19-30.

13. Keage HA, Carare RO, Friedland RP, et al. Population based studies of sporadic cerebral amyloid angiopathy and dementia: a systematic review. BMC Neurol 2009; 9: 3. 
14. Mountjoy CQ, Tomlinson BE, Gibson PH. Amyloid and senile plaques and cerebral blood vessels. J Neurol Sci 1982; 57: 89103.

15. Olichney JM, Hansen LA, Hofstetter CR, et al. Cerebral infarction in Alzheimer's disease is associated with severe amyloid angiopathy and hypertension. Arch Neurol 1995; 52: 702-708.

16. Vonsattel JP, Myers RH, Hedley-Whyte ET, et al. Cerebral amyloid angiopathy without and with cerebral hemorrhages: a comparative histological study. Ann Neurol 1991; 30: 637 649 .

17. Vinters HV. Cerebral amyloid angiopathy. A critical review. Stroke 1987; 18: 311-324.

18. Grysiewicz R, Goerlick PB. Update on amyloid-associated intracerebral haemorrhage. Eur Neurol Review 2012; 7: 22-24.

19. Karbowniczek A, Wierzba-Bobrowicz T, Mendel T, Nauman P. Cerebral amyloid angiopathy manifestated as a brain tumour. Clinical and neuropathological characteristics of two cases. Folia Neuropathol 2012; 50: 194-200.

20. Mendel T, Bertrand E, Szpak GM, et al. Cerebral amyloid angiopathy as a cause of an extensive brain hemorrhage in adult patient with Down's syndrome - a case report. Folia Neuropathol 2010; 48: 206-211.

21. Mendel T, Bertrand E, Szpak GM, et al. Complications of severe cerebral amyloid angiopathy in the course of dementia with Lewy bodies. A case report. Folia Neuropathol 2010; 48: 293299.

22. Pezzini A, Del Zotto E, Volonghi I, et al. Cerebral amyloid angiopathy: a common cause of cerebral hemorrhage. Curr Med Chem 2009; 16: 2498-2513.

23. Rensink AA, de Waal RM, Kremer B, Verbeek MM. Pathogenesis of cerebral amyloid angiopathy. Brain Res Rev 2003; 43 : 207-223.

24. Szpak GM, Lewandowska E, Sliwińska A, et al. Inflammatory cerebral amyloid angiopathy: the overlap of perivascular (PANlike) with vasculitic (A $\beta$-related angiitis) form: an autopsy case. Folia Neuropathol 2011; 49: 335-347.

25. Jellinger KA. Alzheimer disease and cerebrovascular pathology: an update. J Neural Transm 2002; 109: 813-836.

26. Viswanathan A, Greenberg SM. Intracerebral hemorrhage. Handb Clin Neurol 2008; 93: 767-790.

27. Auriel E, Greenberg SM. The pathophysiology and clinical presentation of cerebral amyloid angiopathy. Curr Atheroscler Rep 2012; 14: 343-350

28. Bell RD, Zlokovic BV. Neurovascular mechanisms and bloodbrain barrier disorder in Alzheimer's disease. Acta Neuropathol 2009; 118: 103-113.

29. Greenberg SM, Briggs ME, Hyman BT, et al. Apolipoprotein E epsilon 4 is associated with the presence and earlier onset of hemorrhage in cerebral amyloid angiopathy. Stroke 1996; 27 : 1333-1337.

30. Greenberg SM, Vonsattel JP, Segal AZ, et al. Association of apolipoprotein E $\varepsilon 2$ and vasculopathy in cerebral amyloid angiopathy. Neurology 1998; 50: 961-965.

31. Revesz T, Ghiso J, Lashley T, et al. Cerebral amyloid angiopathies: a pathologic, biochemical, and genetic view. J Neuropathol Exp Neurol 2003; 62: 885-898

32. Marín-Padilla M, Knopman DS. Developmental aspects of intracerebral microvasculature and perivascular spaces: insight into brain response to late-life diseases. J Neuropathol Exp Neurol 2011; 70: 1060-1069.

33. Mendel T, Gromadzka G. Apolipoprotein E (APOE) gene polymorphism and risk and prognosis in cerebral amyloid angiopathyrelated haemorrhage. Neurol Neurochir Pol 2010; 46: 591-597.

34. Hamilton NB, Attwell D, Hall CN. Pericyte-mediated regulation of capillary diameter: a component of neurovascular coupling in health and disease. Front Neuroenergetics 2010; 2: 1-14.

35. Kumar-Singh S. Cerebral amyloid angiopathy: pathogenetic mechanisms and link to dense amyloid plaques. Genes Brain Behav 2008; 7 Suppl 1: 67-82.
36. Lee JM, Yin K, Hsin I, et al. Matrix metalloproteinase-9 in cerebral-amyloid-angiopathy-related hemorrhage. J Neurol Sci 2005; 229-230: 249-254.

37. Attems J, Jellinger K, Thal DR, Van Nostrand W. Review: sporadic cerebral amyloid angiopathy. Neuropathol Appl Neurobiol 2011; 37: 75-93.

38. Zhu GM, Zhang WW, Liu Y, Li J. Arterioles in cerebral amyloid angiopathy and vascular dementia. Chin Med J 2009; 122 : 2985-2988.

39. Hawkes CA, Härtig W, Kacza J, et al. Perivascular drainage of solutes is impaired in the ageing mouse brain and in the presence of cerebral amyloid angiopathy. Acta Neuropathol 2011; 121: 431-443.

40. Schley D, Carare-Nnadi R, Please CP, et al. Mechanisms to explain the reverse perivascular transport of solutes out of the brain. J Theor Biol 2006; 238: 962-974.

41. Zlokovic BV. Neurovascular mechanisms of Alzheimer's neurodegeneration. Trends Neurosci 2005; 28: 202-208.

42. Carrano A, Hoozemans JJ, van der Vies SM, et al. Neuroinflammation and blood-brain barrier changes in capillary amyloid angiopathy. Neurodegenerative Dis 2012; 10: 329-331.

43. Armstrong RA. Size frequency distribution of $\beta$-amyloid $(A \beta)$ deposits: a comparative study of four neurodegenerative disorders. Folia Neuropathol 2012; 50: 240-249.

44. Christoforidis M, Schober R, Krohn K. Genetic-morphologic association study: association between the low density lipoproteinreceptor related protein (LRP) and cerebral amyloid angiopathy. Neuropathol Appl Neurobiol 2005; 31: 11-19.

45. Miners JS, Baig S, Palmer J, et al. A $\beta$-degrading enzymes in Alzheimer's disease. Brain Pathol 2008; 18: 240-252.

46. Nicoll JA, Burnett C, Love S, et al. High frequency of apolipoprotein $\mathrm{E} \varepsilon 2$ in patients with cerebral hemorrhage due to cerebral amyloid angiopathy. Ann Neurol 1996; 39: 682-683.

47. Ruzali WA, Kehoe PG, Love S. LRP1 expression in cerebral cortex, choroids plexus and meningeal blood vessels: relationship to cerebral amyloid angiopathy and $A P O E$ status. Neurosci Lett 2012; 525: 123-128.

48. Tanskanen M, Myllykangas L, Saarialho-Kere U, Paetau A. Matrix metalloproteinase-19 expressed in cerebral amyloid angiopathy. Amyloid 2011; 18: 3-9.

49. Kanekiyo T, Bu G. LPR1 and cerebral amyloid angiopathy. Future Neurol 2009; 4: 55-65.

50. Alonzo NC, Hyman BT, Rebeck GW, Greenberg SM. Progression of cerebral amyloid angiopathy: accumulation of amyloid- $\beta 40$ in affected vessels. J Neuropathol Exp Neurol 1998; 57: 353-359.

51. Love S, Miners S, Palmer J, et al. Insights into pathogenesis and pathogenicity of cerebral amyloid angiopathy. Front Biosci 2009; 14: 4778-4792.

\section{Address for correspondence}

Tadeusz Andrzej Mendel MD, PhD

Institute of Psychiatry and Neurology

Sobieskiego 9

02-957 Warszawa, Poland

e-mail: mendel@ipin.edu.pl 